\title{
Genetic Mechanisms Underlying Sleep
}

\author{
Hirofumi Toda, Mi Shi, Julie A. Williams, and Amita Sehgal \\ Howard Hughes Medical Institute, Perelman School of Medicine University of Pennsylvania, \\ Philadelphia, Pennsylvania 19104, USA \\ Correspondence: amita@pennmedicine.upenn.edu
}

\begin{abstract}
Sleep is important for cognitive ability, and perturbations of sleep are associated with a myriad of brain disorders. However, how sleep promotes health and function during wake is poorly understood. To address the cellular and molecular mechanisms underlying sleep, we use the fruit fly Drosophila melanogaster as a genetic model. Forward genetic approaches in flies were critical for deciphering molecular mechanisms of the circadian clock. Using similar approaches, we and others are gaining insights into the pathways that control sleep amount.
\end{abstract}

Sleep remains a major mystery of biology. Although we spend one-third of our lives sleeping, we still do not know why we sleep or what makes us sleepy. Disruption of sleep causes cognitive loss (Goel et al. 2009) and is also associated with several neurological and psychiatric disorders (Spira et al. 2014), so sleep is clearly required for optimal brain function, but how it does so is still nebulous. Prolonged wakefulness generates the drive to sleep, but what is the molecular basis of this drive? Currently, the "why" and "what" questions regarding sleep are addressed independently, with the former pertaining to the function of sleep and the latter to the regulation of sleep. However, an understanding of the regulation of sleep drive could provide clues to the function served by sleep.

The neurochemistry of sleep is well-studied, such that wake- and sleep-promoting neuromodulators have been described and their effects on sleep have even been mapped to specific loci in the mammalian brain (Saper et al. 2001). How activity of these modulators is controlled and whether they have a central role in the endogenous regulation of sleep is, nevertheless, unclear. Given the lack of a framework for sleep control, an unbiased approach is perhaps the best way to tackle the problem. Such an approach is facilitated by the use of small animal models that lend themselves to genetic manipulation (Sehgal and Mignot 2011).

\section{USING DROSOPHILA TO UNDERSTAND SLEEP}

Prompted by the successful use of the fruit fly (Drosophila melanogaster) for deciphering the molecular mechanism of the circadian clock (Sehgal 2017b), we sought to develop it as a model to study the molecular and genetic mechanisms of sleep. This required demonstrating that behavioral rest in Drosophila represents a sleep state. We already knew that rest in flies was consolidated largely to the nighttime by the circadian clock and we showed that other behavioral criteria for sleep (Campbell and Tobler 1984) are also met by the fly rest state. In particular, we found that flies resting for 5 or more minutes have an increased arousal threshold, in that they are less sensitive to sensory stimulation, and flies deprived of rest during the night compensate subsequently by undergoing a rest rebound the following morning (Hendricks et al. 2000). Thus, rest in flies is controlled by homeostatic mechanisms, which is a hallmark of sleep (Shaw et al. 2000). The fly model for sleep was adopted by laboratories worldwide and was followed by development of similar models in worms (Caenorhabditis elegans), Aplysia, zebrafish (Danio rerio) and, most recently, in jellyfish (Zhdanova et al. 2001; Raizen et al. 2008; Vorster et al. 2014; Nath et al. 2017). The use of these models is rapidly providing insights into mechanistic aspects of sleep (Keene and Duboue 2018).

Although the small size of Drosophila is not conducive to routine measurements of brain activity, electrophysiological correlates of fly sleep have been found (Nitz et al. 2002; van Swinderen et al. 2004). Also, sleep cycles break down in flies with age, much as they do in mammalian species (Koh et al. 2006; Luo et al. 2012). Importantly, drugs that affect the amount of human sleep have corresponding effects on Drosophila sleep, indicating shared neurochemistry (Hendricks et al. 2000, 2003). Indeed, tests of different neurotransmitter pathways in Drosophila have validated arousal promoting effects of dopamine and octopamine (fly norepinephrine) and sleep promoting effects of GABA (Kume et al. 2005; Agosto et al. 2008; Crocker et al. 2010). Conserved roles are also reported for epidermal growth factor and cAMP signaling (Hendricks et al. 2001; Foltenyi et al. 2007). However, the power of Drosophila lies in the unbiased approaches mentioned above, specifically in the use of forward genetic screens to identify mutants and, optimistically, novel components relevant for a biological process. It was such screens that led to the identification of circadian clock

(C) 2018 Toda et al. This article is distributed under the terms of the Creative Commons Attribution-NonCommercial License, which permits reuse and redistribution, except for commercial purposes, provided that the original author and source are credited. 
molecules and the elucidation of the clock mechanism (Sehgal 2017a,b). Arguably, Drosophila might offer an additional advantage for the isolation of sleep mutants, because their low level of redundancy of genes usually allows for stronger phenotypes across many physiological processes. Unlike circadian rhythms, which are very consistent and stereotypical, sleep tends to be variable and modifiable (e.g., by genetic background, environment, nutrition), which could make analysis of weak phenotypes very difficult. A robust phenotype is required if one is to use mutants to dissect mechanism.

\section{FORWARD GENETIC SCREENS TO ISOLATE SLEEP MUTANTS IN DROSOPHILA}

Not surprisingly, forward genetic screens for sleep mutants were initiated shortly after development of the Drosophila model for sleep. The focus, at first, was on mutants that sleep less over the course of a 24-h day, using the 5-min definition described above. The Cirelli and Tononi laboratories identified a short-sleep phenotype in Shaker mutants, which lack a voltage-gated potassium channel (Cirelli et al. 2005). We discovered a mutant, sleepless (sss), that shows very little sleep and, like Shaker, affects neuronal excitability. Interestingly, sss encodes a smallcell-surface, GPI (glycosyl-phosphatidylinositol)-linked protein that activates the Shaker channel (Koh et al. 2008). Subsequently, through a different screen, we identified another short-sleeping mutant, redeye (rye), whose short-sleep phenotype is caused by a lesion in the $\alpha$ subunit of a nicotinic acetylcholine receptor ( $\mathrm{nAchR}$ ) (Shi et al. 2014). Interestingly, SSS also interacts functionally with RYE and serves to inhibit cholinergic signaling (Shi et al. 2014). Other short-sleeping mutants isolated through forward genetic screens include insomniac and an allele of fumin, both of which affect dopamine signaling, and wideawake, which affects GABA signaling (Wu et al. 2008; Stavropoulos and Young 2011; Pfeiffenberger and Allada 2012; Liu et al. 2014). Cyclin A and TARANIS were also identified as sleep-regulating proteins through forward genetic screens, and although both of these are implicated in cell cycle control, the basis of their sleep effect is not known (Rogulja and Young 2012; Afonso et al. 2015).

Thus, genetic screens in Drosophila are successfully identifying sleep genes whose loss generates strong phenotypes. In fact, we are even identifying interactions between these genes, indicating that on a pathway level these screens could even be considered saturating (note that saturation typically requires isolation of multiple alleles of the same gene). However, most of these genes encode neuromodulators that either affect neural excitability or classical neurotransmitter signaling. The hope that forward genetic screens would identify a novel sleep-regulating pathway has yet to be realized. But this does not mean that these genes are not important. We suggest that they are important determinants of daily sleep amount. Indeed, mammalian/human studies of sleep duration are implicating similar genes. Shaker has conserved effects on sleep in mice, and humans with a neurological syndrome whose symptoms include insomnia have autoantibodies to voltage-gated potassium channels (i.e., the Shaker family) (Cornelius et al. 2011). Moreover, a human genome-wide association study (GWAS) identified single-nucleotide polymorphisms associated with altered sleep duration, and although the study focused on a KATP channel, and also showed effects of this channel on fly sleep, the top hits included a protein that regulates Shaker as well as an $\alpha$ subunit of a nAchR (Allebrandt et al. 2013).

So, the question is: Do these pathways constitute homeostatic signals? Do changes in these pathways account for the buildup of sleep drive and the triggering of sleep? We suggest that these pathways act downstream from the homeostatic signal to allow sleep to occur but are themselves not the trigger. In support of this idea, we find that most of the short-sleeping mutants identified to date are compromised in terms of life span and fitness. Molecular analysis indicates parallels between these mutants and sleep-deprived animals, suggesting that they have the need to sleep but cannot sleep. Finally, where tested, these molecules do not drive sleep when overexpressed. Their loss reduced sleep, but their increased activity does not promote sleep, so they are permissive for sleep but not instructive (Wu et al. 2010; Stavropoulos and Young 2011; Shi et al. 2014).

\section{IDENTIFICATION OF A GENE WHOSE OVEREXPRESSION INDUCES SLEEP}

We sought to identify genes instructive for sleep and, to this end, conducted a gain-of-function screen, a screen for genes whose overexpression would induce sleep (Toda et al. 2019). We used the well-known Gal4-UAS system in Drosophila, employing an RU486-inducible neuronal Gal4 driver to overexpress many genes across the fly genome. Our efforts were facilitated by the availability of thousands of previously generated transgenic lines, each containing an upstream Gal4 target sequence, UAS (upstream activation sequence). We screened more than 12,000 such lines, representing more than 8000 genes, and isolated seven lines that showed consistent changes in sleep. These seven lines mapped to six genes (one gene was identified twice through two independent insertions), and five of these reduced sleep when overexpressed. Thus, only one line showed increased sleep with RU486 induction of the UAS-tagged gene (Fig. 1), and we named this gene nemuri (Japanese word for sleep) (Toda et al. 2019).

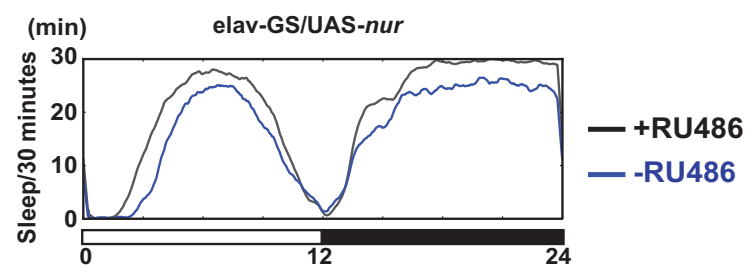

Figure 1. Induction of nur promotes sleep: nur gene was expressed under the control of a panneuronal (elav) RU486-inducible driver, and sleep was monitored in flies treated with RU486 (black) and untreated controls (blue). nur induction by RU486 increased sleep during the day and night. 
The original screen used a beam-break assay to monitor locomotor activity and calculated sleep amount (based on the 5-min definition described above). In follow-up experiments, we confirmed increased sleep upon nemuri (nur) overexpression through video tracking analysis, which is commonly used for higher-resolution recording of sleepwake behavior (Garbe et al. 2015). We found that sleep was not only increased in nur overexpressors but was also deeper, in that the animals were resistant to stimuli that aroused control flies. Circadian rhythms, on the other hand, were normal (Toda et al. 2019).

To address the role of nur in normal sleep regulation, we generated mutants using the CRISPR-Cas9 technique. Analysis of daily sleep in nur mutants did not reveal any changes in sleep amount. However, as opposed to nur overexpressors that are resistant to arousing stimuli, nur mutants were hyperarousable. Both light as well as olfactory stimuli aroused more nur flies than controls. Also, once aroused, the nur flies took longer to go back to sleep (Fig. 2; and data not shown); in other words, their latency to sleep was increased. And finally, the nur mutants showed altered kinetics of recovery sleep following a night of deprivation. That is, the amount of recovery sleep in nur mutants the following morning was maintained, but the recovery was delayed relative to deprived control animals, indicating that the nur mutants have trouble initiating sleep (Toda et al. 2019).

\section{nur ENCODES A SECRETED ANTIMICROBIAL PEPTIDE}

The nur gene encodes a small protein of $\sim 170$ amino acids, which includes a signal sequence (Fig. 3). Lack of a transmembrane region suggested that the signal sequence targets NUR to the membrane for secretion. Indeed, using a cell culture assay, we verified that the NUR protein is secreted. We also showed that secretion of NUR is required for its sleep-inducing properties as expression of a nur transgene lacking the signal sequence failed to increase sleep in flies.

Light pulse for $1 \mathrm{sec}$ at $\mathrm{ZT} 20$

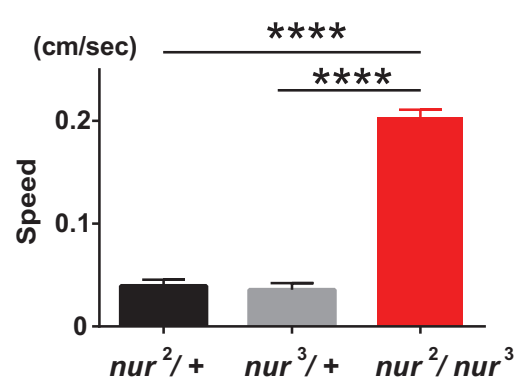

Figure 2. nur mutants are hyperarousable by a light stimulus at night: Flies lacking the nur gene (nur ${ }^{2}$ and $n u r^{3}$ represent two independent isolates of the same CRISPR-induced null mutation) and their heterozygous controls were treated with a 1-sec light pulse at ZT20 ( $8 \mathrm{~h}$ into the night phase). Trans-heterozygous mutants showed increased speed of movement relative to the heterozygotes.

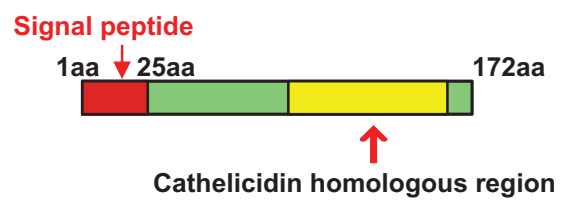

Figure 3. The nur gene encodes a secreted antimicrobial peptide: The schematic of the NUR protein depicts the signal sequence and the region that is homologous to an antimicrobial peptide, Cathelicidin, in cod.

The nature of the peptide encoded by nur was not initially obvious as it did not show homology with any biochemically characterized protein. Based on the high number of basic amino acid residues, which are also found in several antibacterial peptides (AMPs), we narrowed our search to AMPs and found homology with a Cathelicidin in fish (Gadus ogac) (Fig. 3). Tests for antimicrobial activity revealed that NUR is effective in killing different species of bacteria at concentrations considered physiological for AMPs. To determine if NUR kills microbes in flies, we subjected nur overexpressors to bacterial infection and assayed their ability to clear the infecting bacteria. Bacterial load was decreased, and survival was increased in flies overexpressing nur, supporting the idea that nur acts as an AMP in vivo. nur mutants did not show reduced survival upon infection, most likely because other AMPs compensate. Compensatory activity among AMPs has been noticed in the context of other assays and so was not surprising. However, nur mutants were different from controls in terms of their sleep amount following infection. Infection typically increases sleep, which enhances survival and thus is thought to be restorative (Toth and Verhulst 2003; Kuo and Williams 2014). Infection-induced sleep was reduced in nur mutants (Fig. 4).

\section{nur IS EXPRESSED UPON SLEEP DEPRIVATION IN LIMITED BRAIN NEURONS}

To determine the site of nur action relevant for sleep, we inserted a Gal4 transgene into the nur locus using the

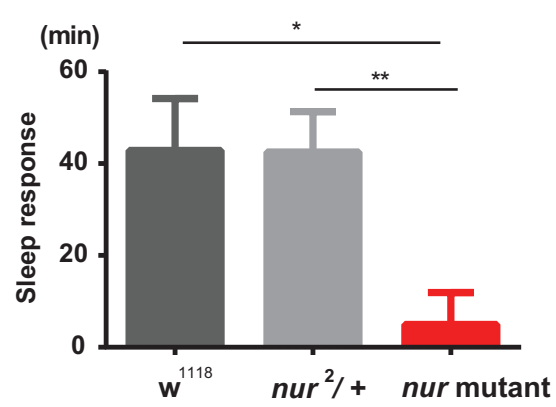

Figure 4. Sleep response after infection with E. coli (ZT0-ZT4). nur mutants are impaired in sleep following bacterial infection: Flies were infected with E. coli $6 \mathrm{~h}$ after lights-off (ZT18) and sleep was monitored the following morning (ZT0-4). Wild-type $\left(w^{1118}\right)$ controls and nur heterozygotes showed increased sleep relative to sleep at this time before infection. The sleep response was abrogated in nur mutants. 
CRISPR-Cas9 technique (Toda et al. 2019). We then used these nur-Gal4 knock-in flies to assay expression of a GFP reporter driven by UAS sequences to identify regions of nur expression in the brain. Early efforts to visualize expression were unsuccessful, leading us to hypothesize that nur is not expressed in the brain. We became aware though that the transcript corresponding to nur was identified previously in the course of a screen for stress-induced genes. Given that sleep deprivation is also a kind of stress, we asked if nur was induced in response to sleep loss. Indeed, we found that, although nur transcript levels were very low in heads of unperturbed flies, sleep deprivation produced a dramatic elevation of nur expression. Thus, we crossed nur ${ }^{\text {Gal4 }}$ with td-GFP, which provides more sensitivity of detection than the standard GFP, and conducted histochemical analysis of brains in sleep-deprived flies. We found the expression of nur in a limited number of cells (as few as one on each side of the brain) that project across the midline. We then assayed expression of NUR protein using an antibody we generated. NUR was detected in the dorsal fan-shaped body (dFSB), a well-characterized sleep-promoting region of the fly brain (Fig. 5; Donlea et al. 2011; Pimentel et al. 2016).

To determine whether expression of NUR in the dFSB is relevant for sleep, we asked whether expression in this structure correlates with the ability of transgenic NUR to drive sleep. A screen of more than 150 Gal4 drivers identified approximately 50 that induce sleep when overexpressing nur. We assayed expression of approximately 10 drivers (selected for their relatively sparse expression) from this entire collection and found those that induced sleep expressed NUR in areas that project to the dFSB. Conversely, those that were negative for sleep induction were also negative for associations with the dFSB. We also determined if the known sleep-promoting neurons that project to the dFSB (the dFSB is a neuropil structure) are targets of NUR. Based on expression of synaptic markers, projections of sleep-promoting dFSB neurons do not appear to be postsynaptic to nur neurons. Also, silencing of these dFSB neurons does not block sleep induction by NUR, indicating that they do not mediate effects of NUR on sleep. More likely, dFSB and NUR neurons target a common downstream locus.

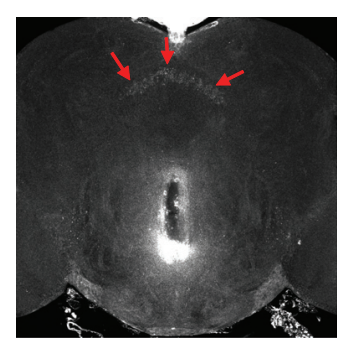

Figure 5. NUR Ab stain in sleep-deprived animal's brain. NUR accumulates in the dorsal fan-shaped body (dFSB) in sleep-deprived flies: Flies were deprived of sleep for $12 \mathrm{~h}$ during the night, following which brains were dissected and stained with NUR antibodies. The arrows point to NUR expression in the dFSB.
Our working model for NUR function is that it has a dual function as an antimicrobial peptide. It kills microbes in the periphery, and perhaps also in the brain, and it promotes sleep through its expression in the brain. The latter is indicated by the fact that NUR overexpression in the fat body (fly equivalent of liver) does not induce sleep. Interestingly, several AMPs are now associated with two functions, but usually both involve the immune system. For instance, an AMP may kill microbes and also attract macrophages to the site of infection (Diamond et al. 2009). Recently, AMPs were reported to play an important role in memory formation, with functions in both brain and fat body (Barajas-Azpeleta et al. 2018). With NUR, we suggest that the second function is to promote survival by increasing sleep. NUR affects arousability during baseline sleep, but an important aspect of its function is to induce sleep following stress such as sleep deprivation and infection. These findings support previous studies of increased immune markers (e.g., proinflammatory cytokines) during sleep loss (Cirelli et al. 2004; Williams et al. 2007; Imeri and Opp 2009; Krueger et al. 2011) and provide a mechanistic link between sleep and immune function. They suggest that sleep following prolonged wakefulness shares regulatory mechanisms with sleep during sickness.

\section{REFERENCES}

Afonso DJ, Liu D, Machado DR, Pan H, Jepson JE, Rogulja D, Koh K. 2015. TARANIS functions with Cyclin A and Cdk1 in a novel arousal center to control sleep in Drosophila. Curr Biol 25: 1717-1726. doi:10.1016/j.cub.2015.05.037

Agosto J, Choi JC, Parisky KM, Stilwell G, Rosbash M, Griffith LC. 2008. Modulation of $\mathrm{GABA}_{\mathrm{A}}$ receptor desensitization uncouples sleep onset and maintenance in Drosophila. Nat Neurosci 11: 354-359. doi:10.1038/nn2046

Allebrandt KV, Amin N, Müller-Myhsok B, Esko T, Teder-Laving M, Azevedo RV, Hayward C, van Mill J, Vogelzangs N, Green $\mathrm{EW}$, et al. 2013. A $\mathrm{K}_{\mathrm{ATP}}$ channel gene effect on sleep duration: from genome-wide association studies to function in Drosophila. Mol Psychiatry 18: 122-132. doi:10.1038/mp.2011 .142

Barajas-Azpeleta R, Wu J, Gill J, Welte R, Seidel C, Mckinney S, Dissel S, Si K. 2018. Antimicrobial peptides modulate longterm memory. PLoS Genet 14: e1007440. doi:10.1371/journal .pgen. 1007440

Campbell SS, Tobler I. 1984. Animal sleep: a review of sleep duration across phylogeny. Neurosci Biobehav Rev 8: 269300. doi:10.1016/0149-7634(84)90054-X

Cirelli C, Gutierrez CM, Tononi G. 2004. Extensive and divergent effects of sleep and wakefulness on brain gene expression. Neuron 41: 35-43. doi:10.1016/S0896-6273(03)00814-6

Cirelli C, Bushey D, Hill S, Huber R, Kreber R, Ganetzky B, Tononi G. 2005. Reduced sleep in Drosophila Shaker mutants. Nature 434: 1087-1092. doi:10.1038/nature03486

Cornelius JR, Pittock SJ, McKeon A, Lennon VA, Aston PA, Josephs KA, Tippmann-Peikert M, Silber MH. 2011. Sleep manifestations of voltage-gated potassium channel complex autoimmunity. Arch Neurol 68: 733-738. doi:10.1001/arch neurol.2011.106

Crocker A, Shahidullah M, Levitan IB, Sehgal A. 2010. Identification of a neural circuit that underlies the effects of octopamine on sleep:wake behavior. Neuron 65: 670-681. doi:10 .1016/j.neuron.2010.01.032

Diamond G, Beckloff N, Weinberg A, Kisich KO. 2009. The roles of antimicrobial peptides in innate host defense. Curr Pharm Des 15: 2377-2392. doi:10.2174/138161209788682325 
Donlea JM, Thimgan MS, Suzuki Y, Gottschalk L, Shaw PJ. 2011. Inducing sleep by remote control facilitates memory consolidation in Drosophila. Science 332: 1571-1576. doi:10.1126/science.1202249

Foltenyi K, Greenspan RJ, Newport JW. 2007. Activation of EGFR and ERK by rhomboid signaling regulates the consolidation and maintenance of sleep in Drosophila. Nat Neurosci 10: 1160-1167. doi:10.1038/nn1957

Garbe DS, Bollinger WL, Vigderman A, Masek P, Gertowski J, Sehgal A, Keene AC. 2015. Context-specific comparison of sleep acquisition systems in Drosophila. Biol Open 4: 15581568. doi:10.1242/bio.013011

Goel N, Rao H, Durmer JS, Dinges DF. 2009. Neurocognitive consequences of sleep deprivation. Semin Neurol 29: 320 339. doi:10.1055/s-0029-1237117

Hendricks JC, Finn SM, Panckeri KA, Chavkin J, Williams JA, Sehgal A, Pack AI. 2000. Rest in Drosophila is a sleep-like state. Neuron 25: 129-138. doi:10.1016/S0896-6273(00) 80877-6

Hendricks JC, Williams JA, Panckeri K, Kirk D, Tello M, Yin JC, Sehgal A. 2001. A non-circadian role for cAMP signaling and CREB activity in Drosophila rest homeostasis. Nat Neurosci 4: 1108-1115. doi:10.1038/nn743

Hendricks JC, Kirk D, Panckeri K, Miller MS, Pack AI. 2003. Modafinil maintains waking in the fruit fly Drosophila melanogaster. Sleep 26: 139-146. doi:10.1093/ sleep/26.2.139

Imeri L, Opp MR. 2009. How (and why) the immune system makes us sleep. Nat Rev Neurosci 10: 199-210. doi:10 $.1038 / \mathrm{nrn} 2576$

Keene AC, Duboue ER. 2018. The origins and evolution of sleep. $J$ Exp Biol 221: jeb159533. doi:10.1242/jeb.159533

Koh K, Evans J, Hendricks J, Sehgal A. 2006. A Drosophila model for age-associated sleep fragmentation. Proc Natl Acad Sci 103: 13843-13847. doi:10.1073/pnas.0605903103

Koh K, Joiner WJ, Wu MN, Yue Z, Smith CJ, Sehgal A. 2008. Identification of SLEEPLESS, a sleep-promoting factor. Science 321: 372-376. doi:10.1126/science.1155942

Krueger JM, Clinton JM, Winters BD, Zielinski MR, Taishi P, Jewett KA, Davis CJ. 2011. Involvement of cytokines in slow wave sleep. Prog Brain Res 193: 39-47. doi:10.1016/B978-0444-53839-0.00003-X

Kume K, Kume S, Park SK, Hirsh J, Jackson FR. 2005. Dopamine is a regulator of arousal in the fruit fly. $J$ Neurosci 25: 7377-7384. doi:10.1523/JNEUROSCI.2048-05.2005

Kuo TH, Williams JA. 2014. Acute sleep deprivation enhances post-infection sleep and promotes survival during bacterial infection in Drosophila. Sleep 37: 859-869. doi:10.5665/sleep .3648

Liu S, Lamaze A, Liu Q, Tabuchi M, Yang Y, Fowler M, Bharadwaj R, Zhang J, Bedont J, Blackshaw S, et al. 2014. WIDE AWAKE mediates the circadian timing of sleep onset. Neuron 82: 151-166. doi:10.1016/j.neuron.2014.01.040

Luo W, Chen WF, Yue Z, Chen D, Sowcik M, Sehgal A, Zheng X. 2012. Old flies have a robust central oscillator but weaker behavioral rhythms that can be improved by genetic and environmental manipulations. Aging Cell 11: 428-438. doi:10 $.1111 / \mathrm{j} .1474-9726.2012 .00800 . \mathrm{x}$

Nath RD, Bedbrook CN, Abrams MJ, Basinger T, Bois JS, Prober DA, Sternberg PW, Gradinaru V, Goentoro L. 2017. The jellyfish Cassiopea exhibits a sleep-like state. Curr Biol 27: 2984-2990.e3. doi:10.1016/j.cub.2017.08.014

Nitz DA, van Swinderen B, Tononi G, Greenspan RJ. 2002. Electrophysiological correlates of rest and activity in Drosophila melanogaster. Curr Biol 12: 1934-1940. doi:10 .1016/S0960-9822(02)01300-3
Pfeiffenberger C, Allada R. 2012. Cul3 and the BTB adaptor insomniac are key regulators of sleep homeostasis and a dopamine arousal pathway in Drosophila. PLoS Genet 8: e1003003. doi:10.1371/journal.pgen.1003003

Pimentel D, Donlea JM, Talbot CB, Song SM, Thurston AJ, Miesenböck G. 2016. Operation of a homeostatic sleep switch. Nature 536: 333-337. doi:10.1038/nature 19055

Raizen DM, Zimmerman JE, Maycock MH, Ta UD, You YJ, Sundaram MV, Pack AI. 2008. Lethargus is a Caenorhabditis elegans sleep-like state. Nature 451: 569-572. doi:10.1038/ nature 06535

Rogulja D, Young MW. 2012. Control of sleep by cyclin A and its regulator. Science 335: 1617-1621. doi:10.1126/science .1212476

Saper CB, Chou TC, Scammell TE. 2001. The sleep switch: hypothalamic control of sleep and wakefulness. Trends Neurosci 24: 726-731. doi:10.1016/S0166-2236(00)02002-6

Sehgal A. $2017 \mathrm{a}$. Flies come through again, period. J Clin Invest 128: $108-109$. doi:10.1172/JCI97839

Sehgal A. 2017b. Physiology flies with time. Cell 171: 1232 1235. doi:10.1016/j.cell.2017.11.028

Sehgal A, Mignot E. 2011. Genetics of sleep and sleep disorders. Cell 146: 194-207. doi:10.1016/j.cell.2011.07.004

Shaw PJ, Cirelli C, Greenspan RJ, Tononi G. 2000. Correlates of sleep and waking in Drosophila melanogaster. Science 287: 1834-1837. doi:10.1126/science.287.5459.1834

Shi M, Yue Z, Kuryatov A, Lindstrom JM, Sehgal A. 2014. Identification of Redeye, a new sleep-regulating protein whose expression is modulated by sleep amount. eLife 3: e01473. doi:10.7554/eLife.01473

Spira AP, Chen-Edinboro LP, Wu MN, Yaffe K. 2014. Impact of sleep on the risk of cognitive decline and dementia. Curr Opin Psychiatry 27: 478-483. doi:10.1097/YCO.000000000000 0106

Stavropoulos N, Young MW. 2011. insomniac and Cullin-3 regulate sleep and wakefulness in Drosophila. Neuron 72: 964 976. doi:10.1016/j.neuron.2011.12.003

Toda H, Williams JA, Gulledge M, Sehgal A. 2019. A sleepinducing gene, nemuri, links sleep and immune function in Drosophila. Science 363: 509-515. doi:10.1126/science aat 1650

Toth LA, Verhulst SJ. 2003. Strain differences in sleep patterns of healthy and influenza-infected inbred mice. Behav Genet 33: 325-336. doi:10.1023/A:1023402709896

van Swinderen B, Nitz DA, Greenspan RJ. 2004. Uncoupling of brain activity from movement defines arousal states in Drosophila. Curr Biol 14: 81-87. doi:10.1016/j.cub.2003.12 .057

Vorster AP, Krishnan HC, Cirelli C, Lyons LC. 2014. Characterization of sleep in Aplysia californica. Sleep 37: 1453-1463. doi:10.5665/sleep.3992

Williams JA, Sathyanarayanan S, Hendricks JC, Sehgal A. 2007. Interaction between sleep and the immune response in Drosophila: a role for the NFKB relish. Sleep 30: 389-400. doi:10 $.1093 /$ sleep/30.4.389

Wu MN, Koh K, Yue Z, Joiner WJ, Sehgal A. 2008. A genetic screen for sleep and circadian mutants reveals mechanisms underlying regulation of sleep in Drosophila. Sleep 31: 465472. doi:10.1093/sleep/31.4.465

Wu MN, Joiner WJ, Dean T, Yue Z, Smith CJ, Chen D, Hoshi T, Sehgal A, Koh K. 2010. SLEEPLESS, a Ly-6/neurotoxin family member, regulates the levels, localization and activity of Shaker. Nat Neurosci 13: 69-75. doi:10.1038/nn.2454

Zhdanova IV, Wang SY, Leclair OU, Danilova NP. 2001. Melatonin promotes sleep-like state in zebrafish. Brain Res 903: 263-268. doi:10.1016/S0006-8993(01)02444-1 


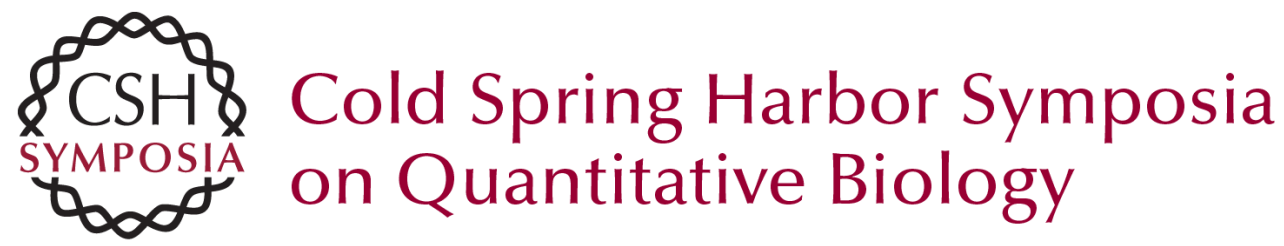

\section{Genetic Mechanisms Underlying Sleep}

Hirofumi Toda, Mi Shi, Julie A. Williams, et al.

Cold Spring Harb Symp Quant Biol 2018 83: 57-61 originally published online April 1, 2019 Access the most recent version at doi:10.1101/sqb.2018.83.037705

References This article cites 48 articles, 9 of which can be accessed free at: http://symposium.cshlp.org/content/83/57.full.html\#ref-list-1
Creative This article is distributed under the terms of the
Commons http://creativecommons.org/licenses/by-nc/4.0/, which permits reuse and License redistribution, except for commercial purposes, provided that the original author and source are credited.

Email Alerting Receive free email alerts when new articles cite this article - sign up in Service the box at the top right corner of the article or click here. 